(1)

CrossMark

\title{
Heat shock protein: a hot topic in idiopathic pulmonary fibrosis
}

\author{
Philippe Bonniaud ${ }^{1,2,3}$, Pierre-Simon Bellaye $e^{2,3,4}$, Olivier Burgy $\mathrm{y}^{2,3}$ and \\ Martin Kolb ${ }^{4}$
}

Affiliations: 'Service de Pneumologie et Soins Intensifs Respiratoires, Centre Hospitalo-Universitaire de Bourgogne, Dijon, France. ${ }^{2}$ Faculté de Médecine et Pharmacie, Université de Bourgogne-Franche Comté, Dijon, France. ${ }^{3}$ NSSERM U866, Dijon, France. ${ }^{4}$ McMaster University, Hamilton, ON, Canada.

Correspondence: Philippe Bonniaud, Service de Pneumologie et Soins Intensifs Respiratoires, Centre Hospitalo-Universitaire de Bourgogne, 21079 Dijon, France. E-mail: philippe.bonniaudachu-dijon.fr

@ERSpublications

HSP90 inhibition could be an exciting new treatment strategy for IPF http://ow.ly/HfKY306uvxw

Cite this article as: Bonniaud P, Bellaye P-S, Burgy O, et al. Heat shock protein: a hot topic in idiopathic pulmonary fibrosis. Eur Respir J 2017; 49: 1602152 [https://doi.org/10.1183/13993003.02152-2016].

Idiopathic pulmonary fibrosis (IPF) is a progressive disease of the lung parenchyma, causing significant morbidity and mortality $[1,2]$. The therapeutic options in IPF are limited to only two recently approved drugs, pirfenidone and nintedanib, which have been shown to slow progression but are not able to stop or reverse the disease [3,4]. Better pathophysiological knowledge is needed to develop new therapeutic strategies in IPF. The current understanding of the disease is that fibroblastic foci, characterised by accumulation of myofibroblasts and overlying "activated" epithelium, represent "hot zones" of the disease and drivers of abnormal extracellular matrix (ECM) accumulation [5]. Transforming growth factor (TGF)- $\beta 1$ is a key cytokine involved in the process of fibrogenesis. TGF- $\beta 1$ causes myofibroblast proliferation and differentiation and increases the synthesis of collagen, fibronectin and many other ECM components [5]. The TGF- $\beta 1$ signalling pathways are complex and occur essentially through serine/ threonine kinase receptors, TGF- $\beta$ receptors type I and II (TGF- $\beta$ RI and TGF- $\beta$ RII). TGF- $\beta$ RII is constitutively active and activates TGF- $\beta$ RI via phosphorylation upon ligand binding [6]. The cytoplasmic proteins Smad 2 and Smad3 predominantly mediate signals from activated TGF- $\beta 1$ receptors. Activation of Smad2 and Smad3 via phosphorylation makes them bind to Smad4, promoting translocation to the nucleus where numerous TGF- $\beta 1$-responsive genes are activated. TGF- $\beta 1$ pathways are undoubtedly very promising but also challenging targets to treat fibrosis and in particular IPF.

In this issue of the European Respiratory Journal, SIBINSKA et al. [7] have nicely demonstrated that heat shock protein (HSP) 90 has a direct role on the TGF- $\beta 1$ signalling pathway and that HSP90 inhibition reduces lung fibrogenesis and fibrosis progression in mice. HSPs, also called stress proteins, were first described as a set of proteins whose expression was (no surprise) induced by rapidly increasing temperature [8]. Like many of science's great discoveries, uncovering the impact of heat on biochemical and molecular systems was an accident. In 1996, Ferrucia Ritossa reflected about his time in the lab in the early 1960s: "I cannot remember whether it was John Pulitzer or Inge or Clara Ghini or Giordano who shifted the temperature of my incubator, but one day I noticed a different puffing pattern". Ritossa worked on Drosophila, which have large chromosomes, and "puffs" in this species are diffused uncoiled regions of DNA that are sites of RNA transcription. HSPs are ubiquitous and highly conserved proteins whose expression is induced in response to a wide panoply of physiological and environmental insults such as heat, oxidative stress or anticancer drugs. Mammalian HSPs have been classified into several families according to their molecular weight: HSP110, HSP90, HSP70, HSP60, HSP47 and small HSPs [9]. Some of

Received: Nov 022016 | Accepted: Nov 042016

Conflict of interest: Disclosures can be found alongside this article at erj.ersjournals.com

Copyright CERS 2017 
them are expressed constitutively whereas others are induced by stressful conditions. Since their discovery, we have learned that the main function of HSPs is to help cells survive conditions that are otherwise lethal. Several mechanisms account for this cytoprotective effect. HSPs are powerful chaperones for other cellular proteins, thus promoting the cellular response to misfolded/aggregated proteins [10]. They participate in the proteasome-mediated degradation of proteins, thereby contributing to the so-called "protein triage" [11]. Furthermore, they act on multiple levels in the apoptotic pathways to ensure that stress-induced damage does not inappropriately trigger cell death [12].

Most of the current knowledge regarding the pathophysiological roles of HSPs has been created in cancer research. These proteins contribute to key phenotypes of cancerous cells, such as uncontrolled proliferation, ability of tissue invasion and induction of angiogenesis. HSPs may facilitate cancer cell survival and thereby become deleterious for cancer patients. Therefore, HSP inhibition has therapeutic appeal and may improve the efficiency of chemotherapy and ultimately disease outcome [13]. The number of HSP inhibitors in pre-clinical evaluation in cancer therapy is rapidly increasing. Specifically, HSP90 inhibitors are currently assessed in combination with chemotherapy for many cancer types, including breast, ovarian, hepatic, colorectal and nonsmall cell lung cancers [14].

The ability of HSPs to modulate cell fate might have important repercussions not only for cancer but also for other progressive disorders such as IPF. Indeed, fibrogenesis and cancer share a number of biological features, including genetic alterations, uncontrolled cell proliferation, epithelial-mesenchymal transition (EMT), altered cell-to-cell communication and tissue invasion [15]. Little is known about the role of HSPs in the initiation and progression of IPF except that HSP47 is a specific chaperone for collagen that has been strongly associated with fibrosis. Among the small HSPs, HSP27 and $\alpha \mathrm{B}$-crystallin have been shown to be involved in renal, vascular and pulmonary fibrosis [16, 17]. The inhibition of HSP27 using OGX-427, an antisense oligonucleotide, protects rodents from subpleural fibrosis induced by TGF- $\beta 1$ overexpression [16]. This compound is currently being tested in clinical trials as a chemo-sensitising agent in cancer therapy. $\alpha \mathrm{B}$-crystallin also has a direct role on the TGF- $\beta 1$ signalling pathway and may be an interesting therapeutic target against IPF [17]. Among the higher molecular weight HSPs, HSP70 probably has an intracellular protective role in pulmonary fibrosis by interfering with Smad proteins but also has pro-inflammatory effects when secreted into the extracellular space [18]. Thus, it seems that the most relevant role for HSPs in fibrogenesis is their ability to modulate the TGF- $\beta 1$ pathway. Interestingly, it has been shown that IPF patients with anti-HSP70 autoantibodies in the circulation have more rapid decline in lung function and higher mortality [19].

SiBINSKA et al. [7] proposed targeting HSP90 for the treatment of IPF. HSP90 family proteins represent 1\% of the total pool of cellular proteins, which makes them the most abundant group of proteins in eukaryotic cells. The HSP90 family is composed of two main members, HSP90 $\alpha$ and HSP90 $\beta$. The distinct role of each isoform remains elusive but HSP90 $\alpha$ and HSP90 $\beta$ have recently been shown to promote cell motility/ differentiation and accelerate wound closure of skin in vivo [20]. HSP90 stabilises actin and tubulin after stress, suggesting a role in the cytoskeletal changes observed during fibrogenesis. In other organs, HSP90 has been attributed a chaperone activity on both TGF- $\beta$ and the glucocorticoid receptor, thus activating fibrotic signalling through TGF- $\beta$ and nuclear factor- $\kappa \mathrm{B}$ pathways [18]. In vitro, HSP90 inhibition totally abrogated TGF- $\beta 1$-induced EMT in lung epithelial cells, and reduced proliferation of myofibroblasts while inducing their apoptosis [18]. SIBINSKA et al. [7] clearly demonstrated that HSP90 is overexpressed in IPF lungs. Hyperplastic type II alveolar epithelial cells next to areas of dense fibrosis showed robust expression of HSP90 $\beta$ compared with control lungs, whereas HSP90 $\alpha$ was absent in these cells. This work suggests that TGF- $\beta$ RII may be a "client protein" of HSP90 $\beta$, thereby promoting receptor stability and enhancing its pro-fibrotic signalling. The authors also showed for the first time that HSP90 inhibition using a water-soluble and orally active inhibitor blocks fibroblast activation and ameliorates bleomycin-induced pulmonary fibrosis in mice.

It is noteworthy that specific inhibitors of HSP90 already exist, which has obviously renewed the interest in these chaperones for clinical testing. The inhibitor used in vivo in this study, 17-DMAG (17-dimethylaminoethylamino-17-demethoxygeldanamycin), a geldanamycin derivative, is a chemical compound that is able to specifically bind the ATP-binding site of HSP90, thereby inhibiting its activity. Animal studies have also highlighted the therapeutic potential of this compound in kidney, liver and skin fibrosis [21]. Other HSP90 inhibitors with novel chemical structure unrelated to geldanamycin are currently being assessed in phase I-III trials for antitumoral properties in combination with chemotherapy.

It is time to explore the feasibility of investigating these new compounds in clinical trials for IPF. They are available for human use and already in advanced clinical stages in cancer. We may not want to miss a golden opportunity and benefit from recent advances in clinical cancer research. A similar success story has been written for the development of nintedanib for IPF, which was originally developed and assessed to treat cancer before the interest was expanded to IPF. Of course a number of critical questions need to 
be clarified first. The most important is the biological impact of durable HSP90 inhibition, considering that HSP90 is ubiquitous and has a role in cell homeostasis under stress conditions and also during physiological processes such as wound healing. What are the consequences of this blockade on other organs? Moreover, it has been shown that HSP90 inhibition may induce other HSPs to counterbalance reduced activity, which may reduce biological and clinical effects in the longer term. Another important question is the clinical safety and efficacy of TGF- $\beta$ signalling inhibition in IPF patients. While most phase I clinical trials involving TGF- $\beta$ inhibitors for cancer have shown that these compounds were relatively well tolerated, long-term exposure and potential side-effects have to be considered.

Nevertheless, there is an ever growing number of clues indicating that targeting HSPs in IPF might become a very hot field in IPF in the near future.

\section{References}

1 Hopkins RB, Burke N, Fell C, et al. Epidemiology and survival of idiopathic pulmonary fibrosis from national data in Canada. Eur Respir J 2016; 48: 187-195.

2 Overgaard D, Kaldan G, Marsaa K, et al. The lived experience with idiopathic pulmonary fibrosis: a qualitative study. Eur Respir J 2016; 47: 1472-1480.

3 Noble PW, Albera C, Bradford WZ, et al. Pirfenidone for idiopathic pulmonary fibrosis: analysis of pooled data from three multinational phase 3 trials. Eur Respir J 2016; 47: 243-253.

4 Richeldi L, Cottin V, du Bois RM, et al. Nintedanib in patients with idiopathic pulmonary fibrosis: combined evidence from the TOMORROW and INPULSIS trials. Respir Med 2016; 113: 74-79.

5 Froese AR, Shimbori C, Bellaye PS, et al. Stretch-induced activation of transforming growth factor- $\beta 1$ in pulmonary fibrosis. Am J Respir Crit Care Med 2016; 194: 84-96.

6 Shi Y, Massagué J. Mechanisms of TGF- $\beta$ signaling from cell membrane to the nucleus. Cell 2003; 113: 685-700.

7 Sibinska Z, Tian X, Korfei M, et al. Amplified canonical transforming growth factor- $\beta$ signalling via heat shock protein 90 in pulmonary fibrosis. Eur Respir J 2017; 49: 1501941.

8 Ritossa FM. Experimental activation of specific loci in polytene chromosomes of Drosophila. Exp Cell Res 1964, 35: 601-607.

9 Kampinga HH, Hageman J, Vos MJ, et al. Guidelines for the nomenclature of the human heat shock proteins. Cell Stress Chaperones 2009; 14: 105-111.

10 Houck SA, Singh S, Cyr DM. Cellular responses to misfolded proteins and protein aggregates. Methods Mol Biol 2012; 832: 455-461.

11 Lanneau D, Wettstein G, Bonniaud P, et al. Heat shock proteins: cell protection through protein triage. ScientificWorldJournal 2010; 10: 1543-1552.

12 Didelot C, Schmitt E, Brunet M, et al. Heat shock proteins: endogenous modulators of apoptotic cell death Handb Exp Pharmacol 2006; 172: 171-198.

13 Jego G, Hazoumé A, Seigneuric R, et al. Targeting heat shock proteins in cancer. Cancer Lett 2013; 332: 275-285.

14 Sidera K, Patsavoudi E. HSP90 inhibitors: current development and potential in cancer therapy. Recent Pat Anticancer Drug Discov 2014; 9: 1-20.

15 Vancheri C. Common pathways in idiopathic pulmonary fibrosis and cancer. Eur Respir Rev 2013; 22: 265-272.

16 Wettstein G, Bellaye PS, Kolb M, et al. Inhibition of HSP27 blocks fibrosis development and EMT features by promoting Snail degradation. FASEB J 2013; 27: 1549-1560.

17 Bellaye PS, Wettstein G, Burgy O, et al. The small heat-shock protein $\alpha \mathrm{B}$-crystallin is essential for the nuclear localization of Smad4: impact on pulmonary fibrosis. J Pathol 2014; 232: 458-472.

18 Bellaye PS, Burgy O, Causse S, et al. Heat shock proteins in fibrosis and wound healing: good or evil? Pharmacol Ther 2014; 143: 119-132.

19 Kahloon RA, Xue J, Bhargava A, et al. Patients with idiopathic pulmonary fibrosis with antibodies to heat shock protein 70 have poor prognoses. Am J Respir Crit Care Med 2013; 187: 768-775.

20 Jayaprakash P, Dong H, Zou M, et al. Hsp90 $\alpha$ and Hsp90 $\beta$ together operate a hypoxia and nutrient paucity stress-response mechanism during wound healing. J Cell Sci 2015; 128: 1475-1480.

21 Tomcik M, Zerr P, Pitkowski J, et al. Heat shock protein 90 (Hsp90) inhibition targets canonical TGF- $\beta$ signalling to prevent fibrosis. Ann Rheum Dis 2014; 73: 1215-1222. 